\title{
Resolving clouds in a global atmosphere model - a multiscale approach with nested models
}

\author{
Fredrik Jansson \\ Centrum Wiskunde \& Informatica \\ Amsterdam, the Netherlands \\ jansson@cwi.nl \\ Daan Crommelin \\ Centrum Wiskunde \& Informatica \\ and University of Amsterdam \\ Amsterdam, the Netherlands
}

\author{
Gijs van den Oord \\ Netherlands eScience Center \\ Amsterdam, the Netherlands
}

\author{
Pier Siebesma \\ KNMI and Delft University of Technology \\ Delft, the Netherlands
}

Index Terms-Atmospheric modeling, superparametrization, multi-scale model

Clouds are challenging to incorporate in global weather and climate models, since they involve processes on length scales much smaller than the typical simulation grid resolution. Therefore clouds are usually not resolved, but represented in a simplified way using so-called parametrization schemes. Accurate cloud modeling is important, since one of the largest remaining uncertainties in climate simulations is related to clouds and the strong effects of cloud feedback on the climate.

We present our project on replacing the cloud parametrization scheme in a global weather model by another smallscale model which can explicitly resolve the cloud processes. This can be seen as a two-way nesting of a high-resolution model inside selected grid columns of the global model. The global weather model in our case is OpenIFS, a version of the ECMWF operational weather forecasting model. The highresolution model is DALES [1], a 3D large-eddy simulation code.

This nesting, also called superparametrization [2], [3], can potentially lead to more accurate simulations, at the cost of requiring more computational power. It also opens the possibility to compare the prediction of the cloud parametrization scheme with the results from the cloud-resolving model and can thus provide a way to validate or improve the cloud parametrizations.

New in our approach is the use of a fully 3D high resolution model for superparametrization, and the possibility of running the high-resolution model in selected grid columns of the global model.

For coupling the two models, we use the OMUSE framework [4]. Both OpenIFS and DALES are written in Fortran. We have created a Python interface to each model using OMUSE. Using these interfaces, both models are controlled from a master script written in Python, which also performs the calculations required for the coupling.

We present results from our superparametrized simulations (see Fig. 1), and discuss the role of small-scale variability for clouds in the superparametrization approach. We also discuss performance measurements for the coupled system, some optimizations, and our experiences with using OMUSE for model coupling.

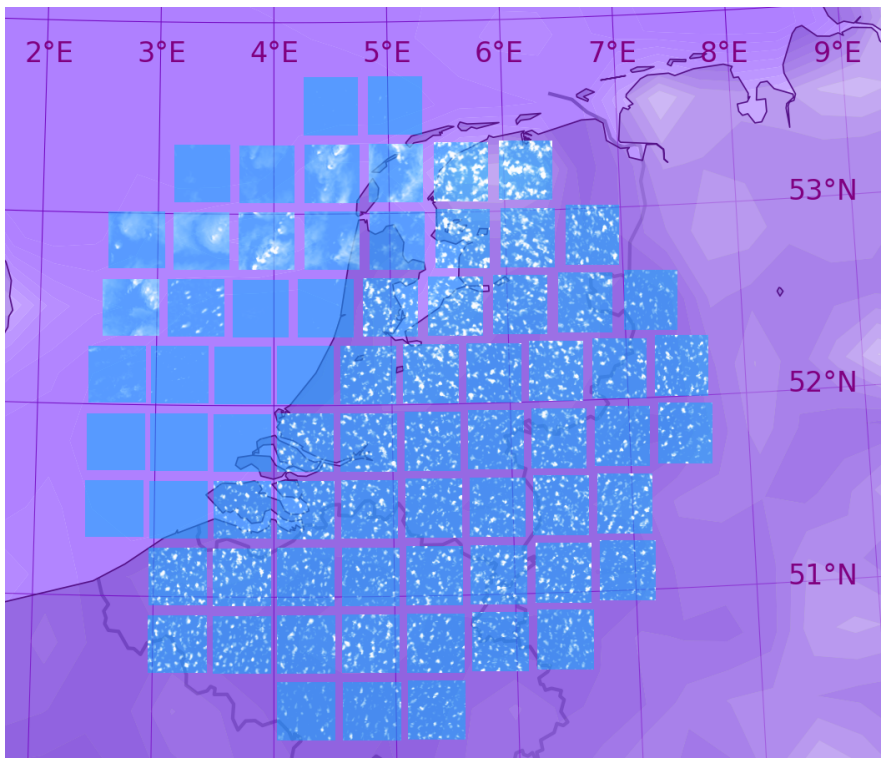

Fig. 1. 72 Large Eddy Simulations (blue boxes) over the Netherlands coupled to OpenIFS (purple background). Cloudiness (liquid water path) is shown in shades of white.

\section{REFERENCES}

[1] T. Heus, C. C. van Heerwaarden, H. J. J. Jonker, A. P. Siebesma, S. Axelsen, K. van den Dries, O. Geoffroy, A. F. Moene, D. Pino, S. R. de Roode, and J. Vilà-Guerau de Arellano, "Formulation of the Dutch Atmospheric Large-Eddy Simulation (DALES) and overview of its applications," GMD, vol. 3, no. 2, pp. 415-444, 2010.

[2] W. W. Grabowski, "An improved framework for superparameterization," J. Atmos. Sci., vol. 61, no. 15, pp. 1940-1952, 2004.

[3] M. Khairoutdinov, D. Randall, and C. DeMott, "Simulations of the atmospheric general circulation using a cloud-resolving model as a superparameterization of physical processes," J. Atmos. Sci., vol. 62, no. 7, pp. 2136-2154, 2005.

[4] I. Pelupessy, B. van Werkhoven, A. van Elteren, J. Viebahn, A. Candy, S. Portegies Zwart, and H. Dijkstra, "The oceanographic multipurpose software environment (OMUSE v1.0)," GMD, vol. 10, no. 8, pp. 3167 3187, 2017. 